\title{
Correlation between Different Helicobacter Morphotypes and Histological Changes in Pig Gastric Mucosa
}

\author{
Nataša Pejčinovska ${ }^{1}$, Dušan Lalošević ${ }^{2}$, Vesna Laloševićc ${ }^{3}$, Ivan Čapo² \& Aleksandar Potkonjak ${ }^{3}$
}

\begin{abstract}
Background: Two distinct morphologic types of bacteria which belong to the Helicobacter species, have been described in pigs: once or twice curved Helicobacter pylori-like bacteria (HLO) and the multicoiled, Gastrospirillum-like bacteria (GLO). The objective of this study was to determine the incidence of Helicobacter spp. using modified Giemsa stain and to define the relationship between presence of Helicobacter spp. and histopathological changes of gastric mucosa in pigs. Materials, Methods \& Results: A total of 120 pig stomachs ( 60 from intensive and 60 from extensive breeding) were enrolled in this study and 240 fragments of fundic and pyloric mucosa were taken for histopathological examination. By modified Giemsa staining of gastric mucosa, Helicobacter-like organisms were confirmed in 4/60 (6.67\%) of pigs in intensive and $5 / 60(8.33 \%)$ of pigs in extensive breeding. The incidence of tightly spiral shaped Gastrospirillum-like organisms in pigs of intensive and extensive breeding were $5 / 60(8.33 \%)$ and $9 / 60(15 \%)$, respectively. The severity of gastritis was scored to the Sydney System with some modifications. There was no significant difference between HLO-positive and HLO-negative fundic mucosa in pigs of both breeding systems. In contrary, there was significant difference between HLO-positive and HLO-negative pyloric mucosa of pigs in intensive $(P<0.001)$ and in extensive breeding $(P<0.05)$. In intensive breeding, there was significant difference between GLO-positive and GLO-negative fundic mucosa $(P<0.05)$, while there was no significant difference between GLO-positive and GLO-negative pyloric mucosa.

Discussion: The prevalence of Helicobacter spp. bacteria were in coherence with literature data. The higher prevalence of both morfological type of bacteria, were considered in pigs in extensive breeding. the hygienic conditions and managment factors in pigs farm are the possible impact for higher bacterial transmission. The association of high prevalence of $H$. pylori and poor hygienic condition was shown by epidemiological studies conducted on humans. In both, humans and pigs, the presence of $H$. pylori correlates with an inflammatory response, but there are differences in inflammatory cell population. In H. pylori infected humans, neutrophils composed the bulk of cellular infiltrate, while in pigs, the primary inflammatory cell was the lymphocyte, which is in accordance to results published by others autors. Thus it indicates that different hosts exhibit a different pathohistological response to the Helicobacter spp. infection. In human as well as in veterinary pathology, the fact of the different pathogenicity of various Helicobacter species is well known. In all HLO-positive pyloric mucosa, moderate to severe focal or diffuse infiltration of mononuclear cells and lymphoid follicles with germinal centers, were observed. A similar conclusion was drawn from results of an experimental infection study in pigs. There was significance between HLO-positive and HLO-negative pyloric mucosa in both, intensive and extensive breeding. In the contrast, GLO were not associated with the presence of severe gastritis, but only with mild to moderate superficial infiltration of lymphocytes and plasma cells in both, fundic and pyloric mucosa. There was no significant difference between GLO-positive and GLO-negative pyloric mucosa of pigs in both breeding systems. Despite the low gastritis score of fundic mucosa in pigs in intensive breeding, there was a significance difference between in GLO-positive and GLO-negative fundic mucosa. It is believed that the possible reason of such results is the meals with low fiber content and low particles size. These results suggest that the presence of HLO, but not of GLO is associated with the pyloric gastritis in pigs.
\end{abstract}

Keywords: Helicobacter, gastritis, histopathology, pigs.

http://dx.doi.org/10.22456/1679-9216.84086

${ }^{1}$ Veterinary Faculty, "St. Kliment Ohridski”, University of Bitola, Bitola, Macedonia. ${ }^{2}$ Faculty of Medicine \& ${ }^{3}$ Department of Veterinary Medicine, Faculty for Agriculture, University of Novi Sad, Novi Sad, Serbia. CORRESPONDENCE: A. Potkonjak [ale @ polj.uns.ac.rs - Tel.: +381 (21) 4853443]. Department of Veterinary Medicine, Faculty of Agriculture, University of Novi Sad. Trg Dositeja Obradovica 8. ZC 21000 Novi Sad, Serbia. 


\section{INTRODUCTION}

Bacteria that colonize the stomach, including curved or S-shaped Helicobacter-like organisms (HLO) and spiral-shaped Gastrospirillum-like organisms (GLO) are isolated from humans $[11,26]$ and several non-human species, including pigs $[1,13,23]$. Infection with $H$. pylori is correlated with histological characteristics of active and chronic gastritis. Chronic gastritis is characterized by increased infiltration of the lamina propria with mononuclear leukocytes [24], and polymorphonuclear neutrophils, which indicates an active component [4]. In both humans and pigs, the infection with $\mathrm{H}$. pylori elicited inflammatory response, but there are differences between inflammatory cell populations. In $H$. pylori infected humans, neutrophils composed the bulk of cellular infiltrate, while in pigs, the primary inflammatory cell was the lymphocyte [3]. The fact of different pathogenicity of different Helicobacter species is well known in veterinary, as well as in human medicine [20]. Helicobacter pylori-like bacteria, characterized as high pathogenic, provoke severe gastritis and lymphoid follicles formation. In contrary, infection with Helicobacter heilmannii, which has been shown to have low pathogenicity, was accompanied by only mild gastritis [14].

The present study was performed with two related aims. The first was to demonstrate the incidence of Helicobacter pylori-like bacteria (HLO) and Gastrospirillum-like bacteria (GLO) in gastric mucosa of pigs. The second goal was to assess possible correlation between different type of Helicobacter spp. (HLO and GLO) and histopathological change of gastric mucosa in pigs.

\section{MATERIALS AND METHODS}

\section{Animals and tissue sampling}

Stomach samples were obtained from 120 apparently healthy pigs ( 60 from intensive and 60 from extensive breeding), 6-7 months old, weighing about $100 \mathrm{~kg}$ and Landrace breed. The stomachs were randomly selected at the slaughterhouse after the carcasses were eviscerated. The stomachs were transported to the laboratory in ice-box $\left(4^{\circ} \mathrm{C}\right)$ and processed within 3 hours after collection. The stomach then were excised along the great curvature, from the diverticulum to pylorus, the contents were discarded and washed gently in tap water, tend to remove only food particles.

\section{Histopathological examination}

Biopsy samples were taken from the fundic and pyloric mucosa and fixed in $10 \%$ neutral formalin for over 24 hours, dehydrated in graded alcohol-xylen series and embedded in paraffin wax. From each block a series of $5 \mu \mathrm{m}$-thick sections were prepared, using a microtome $\left(\text { Leica }^{\circledR}\right)^{1}$. All tissue sections were stained with hematoxylin (Mayers healum solution $\left.{ }^{\circledR}\right)^{2}$ and eosin $\left(\text { Eosyn Y solution }{ }^{\circledR}\right)^{2}$ for histopathologic examination and modified Giemsa stains (Giemsa stain solution $\left.{ }^{\circledR}\right)^{2}$ for detection of bacteria [8]. The severity of gastritis was scored to the updated Sydney System [6] with some modifications, according to Park et al. [19] as: 0 - no infiltration of inflammatory cells, 1-mild infiltration of lymphocytes and plasma cells, 2 - moderate dense infiltration of lymphocytes and plasma cells, but no lymphoid follicles, 3 - moderate dense infiltration of lymphocytes and plasma cells and presence of lymphoid follicles, 4 - very dense infiltration of lymphocytes and plasma cells and presence of lymphoid follicles. Both diffuse infiltration with inflammatory cells and the presence of lymphoid aggregates and lymphoid follicles in the mucosa were taken into consideration. All samples were assessed using the light microscope (Olympus CX31RBSF $\left.{ }^{\circledR}\right)^{3}$.

According to microscopic examination, the stomachs were divided into two groups: Helicobacter spp.-positive: HLO-positive and GLO-positive group and Helicobacter spp.-negative: HLO-negative and GLO-negative group. Based on the scoring of the diffuse infiltration with inflammatory cells and the presence of lymphoid aggregates and lymphoid follicles, an overall gastritis score was obtained. Therefore, the average score of fundic and pyloric mucosa was calculated for each group: HLO-positive and HLO-negative, as well as GLO-positive and and GLO-negative group.

\section{Statistical analyzes}

The statistical analysis was performed by StatSoft Statistica (Version 6.0) ${ }^{4}$. The significant differences between HLO-positive and HLO-negative, as well as GLO-positive and GLO-negative fundic and pyloric mucosa was analyzed by two tailed Students $t$-test. Values of $P<0.05$ were considered significant. All data were shown as mean \pm standard deviation (SD). 
N. Pejčinovska, D. Lalošević, V. Lalošević, I. Čapo \& A. Potkonjak. 2018. Correlation between Different Helicobacter Morphotypes and Histological Changes in Pig Gastric Mucosa.

RESULTS

Detection of Helicobacter-spp. organisms (HLO and GLO)

According to morphological characteristics, Helicobacter-like organisms (HLO) were 1-2 coiled or curved, mainly in the upper half of gastric pits (Figure 1). Microorganisms were not seen in lamina propria or lymphoid follicles. The bacteria were observed in $4(6.67 \%)$ out of a total of 60 stomachs in intensive breeding and $5(8.33 \%)$ out of a total of 60 stomachs

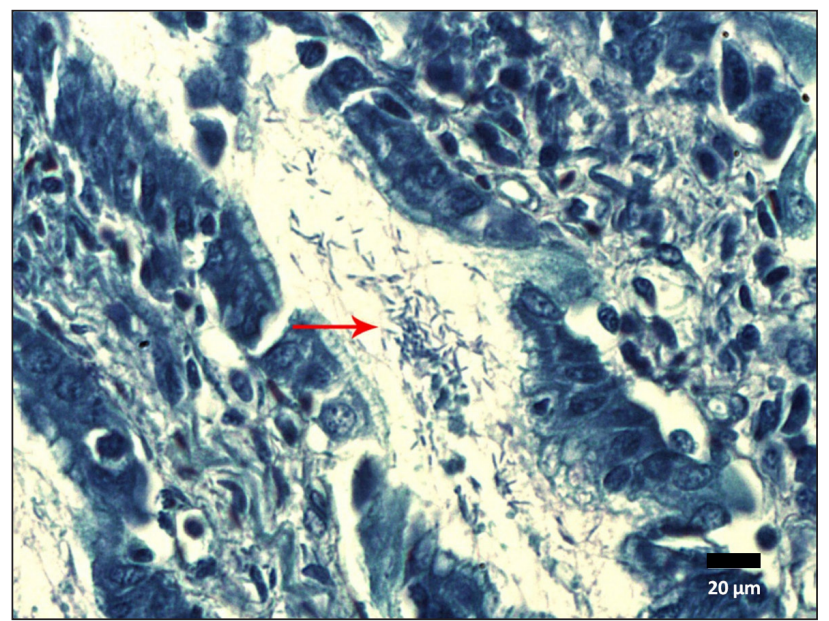

Figure 1. Numerous Helicobacter-like organisms (arrow) in pig gastric pits (modified Giemsa). [bar $=20 \mu \mathrm{m}]$.

\section{Histopathology}

Histopathological evaluation of gastric mucosa of pigs based on presence of different bacteria morphotypes is presented in Table 1 . The percentage of Helicobacter spp. gastritis (in both, HLO and GLO) was higher in gastric mucosa of pigs in extensive breeding than pigs in intensive breeding. In contrary, the percentage of Helicobacter-spp. (HLO/GLO)negative gastritis was higher in stomach mucosa of pigs in intensive breeding, than pigs in extensive breeding.

\section{Histopathologic changes in HLO-positive mucosa}

In the fundic and pyloric mucosa of all infected pigs, were found microscopic lesions specific for $H$. pylori associated gastritis. In the fundic mucosa, the mild inflammatory response was observed as a superficial infiltration of lymphocytes and plasma cells, mainly in upper third of lamina propria, nearby gastric pits. Lymphoid follicles formation close to lamina muscula- in extensive breeding. The spiral shaped Gastrospirillum-like organisms (GLO) were observed in 5 (8.33\%) out of a total of 60 stomachs in intensive breeding and 9 (15\%) out of a total of 60 stomachs in extensive breeding. The bacterium had a peculiar screw-like appearance, easily differentiated from other curved bacteria. Microorganism was observed in gastric pits or deeply in the lumen of gastric glands (Figure 2). The prevalence of Helicobacter spp. bacteria (in both HLO and GLO morphotype) is higher in gastric mucosa of pigs in extensive breeding than pigs in intensive breeding.

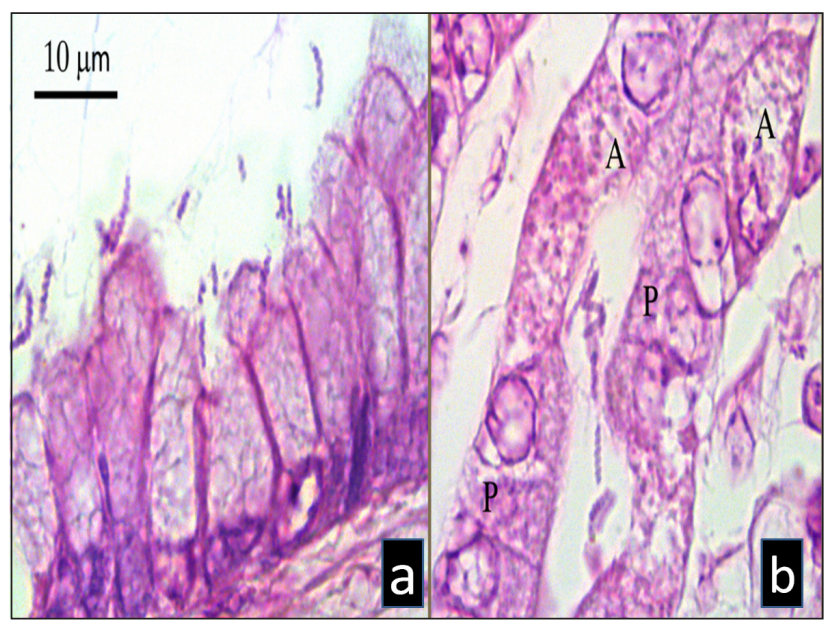

Figure 2. Gastrospirillum-like organisms in the lumen of gastric pit (a) and gland (b), a lack of any leukocytes around, a- Gastric pit, b- GLO deeply in the fundic gland, A- Acidogenic cells, P- Pepsinogenic cells (H\&E). [bar= $10 \mu \mathrm{m}]$.

ris mucosae, were also seen. In intensive breeding, the average gastritis score of the HLO-positive fundic mucosa was $1.5 \pm 0.58$, whereas that of the HLO-negative fundic mucosa was $1.16 \pm 0.37$. In extensive breeding, the average gastritis score of the HLO-positive fundic mucosa was $1.4 \pm 0.55$, whereas that of the HLOnegative fundic mucosa was $1.13 \pm 0.34$. There was no significant difference between HLO-positive and HLO-negative fundic mucosa. In the pyloric mucosa, moderate to severe focal or diffuse infiltration trough the lamina propria by lymphocytes and plasma cells was observed. The inflammatory infiltrate extended from the surface epithelial cells to the lamina muscularis mucosae. Lymphocytes tended to accumulate in small aggregates or confluent foci that matured into lymphoid follicles with germinal centers (Figure 3). Except for lymphocytes, the only other inflammatory cells found in significantly increased number were the plasma cells. In intensive breeding, the average gastritis 
score of the HLO-positive pyloric mucosa was $2 \pm 0.00$, whereas that of the HLO-negative pyloric mucosa was $1.57 \pm 0.50$. There was a significant difference between HLO-positive and HLO-negative pyloric mucosa $(P<0.001)$. In extensive breeding, the average gastritis score of the HLO-positive pyloric mucosa was $2.2 \pm$ 0.45 , whereas that of the HLO-negative pyloric mucosa was $1.38 \pm 0.49$. There was a significant difference between HLO-positive and HLO-negative pyloric mucosa $(P<0.05)$.

Table 1. Histopathological evaluation of gastric mucosa of pigs based on presence of different bacteria morphotypes.

\begin{tabular}{ccccc}
\hline \multirow{2}{*}{ Types of bacterial gastritis } & \multicolumn{2}{c}{ Intensive breeding } & \multicolumn{2}{c}{ Extensive breeding } \\
\cline { 2 - 5 } & Frequency & Percentage & Frequency & Percentage \\
\hline HLO-positive gastritis & 4 & $6.67 \%$ & 5 & $8.33 \%$ \\
GLO-positive gastritis & 5 & $8.33 \%$ & 9 & $15 \%$ \\
Helicobacter spp. (HLO/GLO)-negative gastritis & 37 & $61.67 \%$ & 24 & $40 \%$ \\
Normal mucosa without Helicobacter spp. & 14 & $23.33 \%$ & 22 & $36.67 \%$ \\
\hline Total & 60 & $100 \%$ & 60 & $100 \%$ \\
\hline
\end{tabular}

HLO: Helicobacter pylori-like organisms; GLO: Gastrospirillum-like organisms.

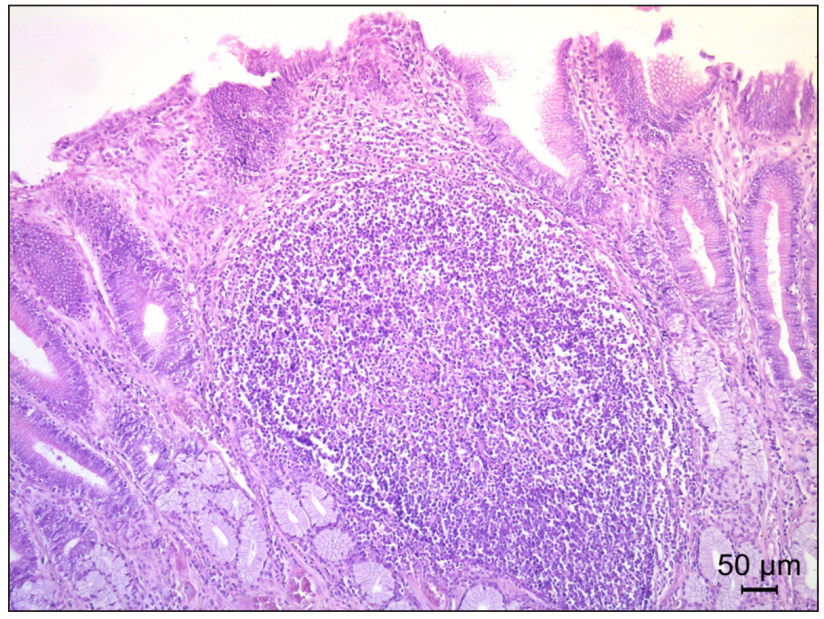

Figure 3. Mild follicular gastritis in the pyloric region (H\&E). [bar=50 $\mu \mathrm{m}]$.

Histopathologic changes in GLO-positive mucosa:

In the fundic mucosa was observed only mild superficial infiltration of lymphocyte and plasma cells, mainly in upper third of lamina propria, nearby surface epithelium. No lymphoid follicles formation, neither lymphoid aggregates, were seen. In intensive breeding, the average gastritis score of the GLO-positive fundic mucosa was $1.0 \pm 0.00$, whereas that of the GLO-negative fundic mucosa was $1.16 \pm 0.37$. There was a significant difference between GLO-positive and GLO-negative fundic mucosa $(P<0.05)$. In extensive breeding, the average gastritis score of the GLO-positive fundic mucosa was $1.0 \pm 0.00$, whereas that of the GLO-negative fundic mucosa was $1.13 \pm 0.34$. There was no significant difference between GLO-positive and GLO-negative fundic mucosa. In the pyloric mucosa, mild to moderate superficial infiltration of lymphocytes and plasma cells was observed in upper third of lamina propria. Presence of lymphoid follicles in the lower third of lamina propria, were also seen. In intensive breeding, the average gastritis score of the GLO-positive pyloric mucosa was $1.4 \pm 0.55$, whereas that of the GLOnegative pyloric mucosa was $1.57 \pm 0.50$. In extensive breeding, the average gastritis score of the GLO-positive pyloric mucosa was $1.22 \pm 0.44$, whereas that of the GLO-negative pyloric mucosa was $1.38 \pm 0.49$. There was no significant difference between GLO-positive and GLO-negative pyloric mucosa in the both group.

\section{DISCUSSION}

It is well known that two main morphologic types of bacteria (tightly spiraled bacteria- Gastrospirillum spp. 
and once or twice curved Helicobacter pylori-like bacteria) can colonize the stomach of pigs $[5,7,13]$. Findings from this study of the prevalence of Helicobacter spp. bacteria are in coherence with literature data. The prevalence of Helicobacter spp. in pig stomachs were reported from $8 \%$ to $10.8 \%[7,19,23]$. The higher prevalence of both morfological type of bacteria, were considered in pigs in extensive breeding. Presumbly, the hygienic conditions and managment factors in pigs farm are the possible impact for higher bacterial transmission. The association of high prevalence of $H$. pylori and poor hygienic condition was shown by epidemiological studies conducted on humans [2]. Also, several factors influenced the infection rate in pigs, such as type of breed [7,23], stress conditions, co-infection with other organisms or even the sensitivity of such detection methods [17].

Gastritis is the result of a natural infection with H. pylori in humans [26] and conventional pigs [13] or an experimental induced infection in gnotobiotic piglets [3,14,15], miniature pigs [12] and conventional piglets [22]. In both, humans and pigs, the presence of $H$. pylori correlates with an inflammatory response, but there are differences in inflammatory cell population. The early stage of $H$. pylori infection in piglets is characterized by neutrophilic infiltrate, which is quickly followed by lymphocytic infiltration [15]. Results of this study indicated that that mononuclear cells (lymphocytes and plasma cells) were predominant cell types of inflammatory infiltrate, whereas polymorphonuclear leukocytes (neutrophils) were not present. Previous study also point to lack of neutrophile in inflammatory infiltrate of experimentally induced H. pylori infection in conventional piglets [22]. This is in accordance to results published by other autors $[1,3,15,22]$, but contrary to chronic active gastritis associated with Helicobacter spp. in humans [9]. Thus it indicates that different hosts exhibit a different pathohistological response to the Helicobacter spp. infection [21]. Signs of gastric inflammation, such as lymphocytic and plasmacytic inflammatory cell infiltrates and lymphoid follicle formation, are most prominent in $H$. pylori-inoculated pigs [14]. A similar conclusion was drawn from results of an experimental infection study in: gnotobiotic pigs [15], miniature pigs [12] and conventional pigs [13,22]. Results for histopathological examination revealed a moderate to severe focal or diffuse lymphoplasmacytic inflammation and lymphoid follicles with germinal centers in all HLO-positive pyloric mucosa, which corresponds to the results of an experimental study [19]. Gastritis in the glandular region was found to be associated with tightly spiraled bacterial infection by several researchers $[7,17,19]$. In this study no correlation was evident between pyloric gastritis and Gastrospirillum spp. infection. Results of this study are consistent with the research of other authors [1,25] which could not find an association between these two parameters. Previously was suggested that Gastrospirillum spp. does not seem to elicit gastric inflammatory disease in pigs [1]. However, despite the low gastritis score of fundic mucosa in pigs in intensive breeding, there was a significance difference between in GLO-positive and GLO-negative fundic mucosa. It is believed that the possible reason of such results is the type of animal nutrition. Furthermore, the meals with low fiber content and low particles size lead to an increased risk of damage to gastric mucosa, especially in animals under stress condition [18]. In most of the GLO-positive pyloric mucosa, the lymphoid aggregates and follicles were observed in the basal portion of lamina propria. Lymphoid aggregates and lymphoid follicles formation in the lamina propria are a common finding in pigs infected with Helicobacter spp. [16]. Strong correlation between "G. suis" and the formation of lymphoid follicles was also reported previously [17].

In humans as well as veterinary pathology, the fact of different pathogenicity of difeerent Helicobacter spp. is well known [20]. The results of current study revealed the higher gastritis score and signifficance between HLO-positive and HLO-negative pyloric mucosa in both, intensive and extensive breeding. In contrary, lower gastritis score and no significant difference between GLO-positive and GLO-negative pyloric mucosa was found in pigs of both breeding systems. These findings indicated that infection of pigs with different Helicobacter species can affect gastric mucosa in various manners and various pathogenic effects of different, but related microorganisms are possible. Helicobacter pylori-like bacteria characterised as high pathogenic, has been associated with severe gastritis and formation of lymphoid follicles [14]. In contrast, infection with Helicobacter heilmannii, which has been shown to have low pathogenicity [10] was accompanied by only mild and limited gastric inflammation [14]. 


\section{CONCLUSIONS}

The results of present study showed that two distinct morphologic types of bacteria (Helicobacter pylori morphotype and Gastrospirillum morphotype) which belong to the Helicobacter species are existed in pigs. Helicobacter spp. in gastric mucosa of pigs which belong to different genus, differ significantly in both, pathogenicity and virulence. Obviously, Gastrospirillum-like morphotype does not seem to elicit gastric mucosal inflammatory response, but Helicobacter pylori-like morphotype act as contributing factor to development of pathological changes in the pyloric mucosa of pigs. Therefore, histopathological examination remains the gold standard for diagnosis of gastritis, which will provide useful information that can enhance nutrition and health status of pigs.

\section{MANUFACTURERS}

${ }^{1}$ Leica Biosystems Inc. Nussloch, Germany.

${ }^{2}$ Merck KGaA. Darmstadt, Germany.

${ }^{3}$ Olympus Corporation. Tokyo, Japan.

${ }^{4}$ StatSoft. Tulsa, OK, USA.

Acknowledgements. This research is in part funding by Grant No.TR31084, Serbian Ministry of Education, Science and Technological Development.

Declaration of interest. The authors report no conflicts of interest. The authors alone are responsible for the content and writing of the paper.

\section{REFERENCES}

1 Barbosa A.J., Silva J.C., Nogueira A.M., Paulino E. \& Miranda C.R. 1995. Higher incidence of Gastrospirillum spp. in swine with gastric ulcer of the pars oesophagea. Veterinary Pathology. 32(2): 134-139.

2 Bardhan P.K. 1997. Epidemiological features of Helicobacter pylori infection in developing countries. Clinical Infectious Diseases. 25(5): 973-978.

3 Bertram T.A., Krakowka S. \& Morgan D.R. 1991. Gastritis associated with infection by Helicobacter pylori: Comparative pathology in humans and swine. Reviews of Infectious Diseases. 13(8): 714-722.

4 Bodger K. \& Crabtree J.E. 1998. Helicobacter pylori and gastric inflammation. British Medical Bulletin. 54(1): 139150.

5 De Groote D., Ducatelle R., Van Doorn L.J., Tilmant K., Verschuuren A. \& Haesebrouck F. 2000. Detection of "Candidatus Helicobacter suis" in gastric samples of pigs by PCR: comparison with other invasive diagnostic techniques. Journal of Clinical Microbiology. 38(3): 1131-1135.

6 Dixon M.F., Genta R.M., Yardley J.H. \& Correa P. 1996. Classification and grading of gastritis: the updated Sydney system. The American Journal of Surgical Pathology. 20(10): 1161-1181.

7 Grasso G.M., Ripabelli G., Sammarco M.L., Ruberto A. \& Iannitto G. 1996. Prevalence of Helicobacter-like organisms in porcine gastric mucosa: A study of swine slaughtered in Italy. Comparative Immunology, Microbiology \& Infectious Diseases. 19(3): 213-217.

8 Gray S.F., Wyatt J.I. \& Rathbone B.J. 1986. Simplified techniques for identifying Campylobacter pyloridis. Journal of Clinical Pathology. 39(11): 1279-12780.

9 Hassall E. \& Dimmick J.E. 1991. Unique feature of Helicobacter pylori disease in children. Digestive Diseases and Science. 36(4): 17-23.

10 Hellemans A., Chiers K., De Bock M., Decostere A., Haesebrouck F., Ducatelle R. \& Maes D. 2007. Prevalence of Candidatus Helicobacter suis in pigs of different ages. The Veterinary Record. 161(6): 189-192.

11 Joosten M., Flahou B., Meyns T., Smet A., Arts J., Cooman L., Pasmans F., Ducatelle R. \& Haesebrouck F. 2013. Case report: Helicobacter suis infection in a pig veterinarian. Helicobacter. 18(5): 392-396.

12 Koga T., Shimada Y., Sato K., Takahashi K., Kikuchi, Miura T., Takenouchi T., Narita T. \& Iwata M. 2002. Experimental Helicobacter pylori gastric infection in miniature pigs. Journal of Medical Microbiology. 51: 238-246.

13 Krakowka S., Ringler S.S., Flores J., Kearns R.J., Eaton K.A. \& Ellis J.A. 2005. Isolation and preliminary characterization of a novel Helicobacter species from swine. American Journal of Veterinary Research. 66(6): 938-944.

14 Krakowka S., Rings D.M. \& Ellis J.A. 2005. Experimental induction of bacterial gastritis and gastric ulcer disease in gnotobiotic swine inoculated with porcine Helicobacter-like species. American Journal of Veterinary Research. 66(6): 945-952. 
15 Krakowka S.T., Morgan D.R., Kraft W.G. \& Leunk R.D. 1987. Establishment of gastric Campylobacter pylori infection in the neonatal gnotobiotic piglet. Infection and Immunity. 55(11): 2789-2796.

16 Mall A.S., Suleman N., Taylor K., Kidd M., Tyler M., Lotz Z., Hickman R. \& Kahn D. 2004. The relationship of a Helicobacter heilmannii infection to the mucosal changes in abattoir and laboratory pig stomach. Surgery Today. 34(11): 943-949.

17 Mendes E.N., Queiroz D.M., Rocha G.A., Nogueira A.M., Carvalho A.C., Lage A.P. \& Barbosa A.J. 1991. Histopathological study of porcine gastric mucosa with and without a spiral bacterium ("Gastrospirillum suis"). Journal of Medical Microbiology. 35: 345-348.

18 Millet S., Kumar S., De Boever J., Meyns T., Aluwe M., De Brabander D. \& Ducatelle R. 2012. Effect of particle size distribution and dietary crude fibre content on growth performance and gastric mucosa integrity of growing-finishing pigs. The Veterinary Journal. 192: 316-321.

19 Park J.H., Lee B.J., Lee Y.S. \& Park J.H. 2000. Association of tightly spiraled bacterial infection and gastritis in pigs. The Journal of Veterinary Medical Science. 62: 725-729.

20 Peyrol S., Lecoindre P. \& Berger I. 1998. Differential pathogenic effect of two helicobacter-like organisms in dog's gastric mucosa. Journal of Submicroscopic Cytology and Pathology. 30: 425-433.

21 Pirarat N., Sada V., Wangnaitham S. \& Sunyasootcharee B. 2007. Pathological Study of Helicobacter spp. infection in pig stomachs. The Thai Journal of Veterinary Medicine. 37(1): 41-48.

22 Poutahidis T., Tsangaris T., Kanakoudis G., Vlemmas I., Iliadis N. \& Sofianou D. 2001. Helicobacter pylori-induced gastritis in experimentally infected conventional piglets. Veterinary Pathology. 38: 667-678.

23 Queiroz D.M., Rocha G.A., Mendes E.N., Lage A.P., Carvalho A.C. \& Barbosa A.J. 1990. A spiral microorganism in the stomach of pigs. Veterinary Microbiology. 24(2): 199-204.

24 Rauws E.A.J., Langenberg W., Houthoff H.J., Hendrik J., Houthoff H., Zanen C., Guido N. \& Tytgat J. 1988. Campylobacter pyloridis associated chronic active antral gastritis. Gastroenterology. 94: 33-40.

25 Roosendaal R., Vos J.H., Roumen T., van Vugt R., Cattoli G., Bart A., Klaasen H.L., Kuipers E.J., VandenbrouckeGrauls C.M. \& Kusters J.G. 2000. Slaughter pigs are commonly infected by closely related but distinct gastric ulcerative lesion-inducing gastrospirilla. Journal of Clinical Microbiology. 38(7): 2661-2664.

26 Warren J.D. \& Marshall B.J. 1983. Unidentified curved bacilli on gastric epithelium in active chronic gastritis. The Lancet. 321(8336): 1273-1275. 